\title{
The Alon-Tarsi number of planar graphs without cycles of lengths 4 and $l$
}

\author{
Huajing $\mathrm{Lu}^{*} \quad$ Xuding $\mathrm{Zhu}{ }^{\dagger \dagger}$
}

September 1, 2021

\begin{abstract}
This paper proves that if $G$ is a planar graph without 4-cycles and $l$-cycles for some $l \in\{5,6,7\}$, then there exists a matching $M$ such that $A T(G-M) \leq 3$. This implies that every planar graph without 4 -cycles and $l$-cycles for some $l \in\{5,6,7\}$ is 1 -defective 3 -paintable.
\end{abstract}

Keywords: planar graph; choice number; paint number; Alon-Tarsi number.

\section{Introduction}

Assume $G$ is a graph and $d$ is a non-negative integer. A $d$-defective coloring of $G$ is a coloring of the vertices of $G$ such that each color class induces a subgraph of maximum degree at most $d$. A 0 -defective coloring of $G$ is also called a proper coloring of $G$. In a coloring of the vertices of $G$, we say an edge $e$ is a fault edge if the end vertices of $e$ receive the same color. A coloring of $G$ is 1-defective if and only if the set of fault edges is a matching. A $k$-list assignment of a graph $G$ is a mapping $L$ which assigns to each vertex $v$ a set $L(v)$ of $k$ permissible colors. Given a $k$-list assignment $L$ of $G$, a $d$-defective $L$-coloring of $G$ is a $d$-defective coloring $c$ of $G$ with $c(v) \in L(v)$ for every vertex $v$ of $G$. A graph $G$ is $d$-defective $k$-choosable if for any $k$-list assignment $L$ of $G$, there exists a $d$-defective $L$-coloring of $G$. We say $G$ is $k$-choosable if $G$ is 0-defective $k$-choosable. The choice number $\operatorname{ch}(G)$ of $G$ is the minimum $k$ for which $G$ is $k$-choosable.

Defective list coloring of planar graphs has been studied in a few papers. Eaton and Hall [4, and Škrekovski [12] proved independently that every planar graph is 2-defective 3-choosable. Cushing and Kierstead [2] proved

\footnotetext{
${ }^{*}$ Department of Mathematics, Zhejiang Normal University, China. e-mail: huajinglu@zjnu.edu.cn

${ }^{\dagger}$ Department of Mathematics, Zhejiang Normal University, China. e-mail: xdzhu@zjnu.edu.cn

${ }^{\ddagger}$ Grant Numbers: NSFC 11571319 and 111 project of Ministry of Education of China.
} 
that every planar graph is 1-defective 4-choosable. The above results can be reformulated as follows:

Assume $G$ is a planar graph. (1) For every 3-list assignment $L$ of $G$, there is a subgraph $H$ of $G$ with $\Delta(H) \leq 2$ and $G-E(H)$ is $L$-colorable. (2) For every 4-list assignment $L$ of $G$, there is a subgraph $H$ of $G$ with $\Delta(H) \leq 1$ and $G-E(H)$ is $L$-colorable.

In the proofs of [2], 44 and [12], the subgraph $H$ depends on the list assignment $L$. A natural question is whether there is a subgraph $H$ that does not depend on $L$. In other words, we ask the following questions:

(1) Is it true that every planar graph $G$ has a subgraph $H$ with $\Delta(H) \leq 2$ such that $G-E(H)$ is 3 -choosable?

(2) Is it true that every planar graph $G$ has a subgraph $H$ with $\Delta(H) \leq 1$, such that $G-E(H)$ is 4-choosable?

It turns out that the answer to (1) is negative and the answer to (2) is positive. Very recently, it is shown in [9] that there is a planar graph $G$ such that for any subgraph $H$ of $G$ with $\Delta(H) \leq 3$ (this number 3 is not a typo), $G-E(H)$ is not 3 -choosable. On the other hand, as a consequence of the main result in [6], every planar graph $G$ has a matching $M$ such that $G-M$ is 4 -choosable.

The main result in [6] is about the Alon-Tarsi number of $G-M$. We associate to each vertex $v$ of $G$ a variable $x_{v}$. The graph polynomial $P_{G}(\mathbf{x})$ of $G$ is defined as $P_{G}(\mathbf{x})=\prod_{u \sim v, u<v}\left(x_{v}-x_{u}\right)$, where $\mathbf{x}=\left\{x_{v}: v \in V(G)\right\}$ and $<$ is an arbitrary fixed ordering of the vertices of $G$. It is easy to see that a mapping $\phi: V \rightarrow R$ is a proper coloring of $G$ if and only if $P_{G}(\phi) \neq 0$, where $P_{G}(\phi)$ means to evaluate the polynomial at $x_{v}=\phi(v)$ for $v \in V(G)$. Thus to find a proper coloring of $G$ is equivalent to find an assignment of $\mathbf{x}$ so that the polynomial evaluated at the assignment is non-zero.

For a mapping $\eta: V(G) \rightarrow\{0,1, \ldots\}$, let $c_{P_{G}, \eta}$ be the coefficient of the monomial $\prod_{v \in V(G)} x_{v}^{\eta(v)}$ in the expansion of $P_{G}$. It follows from the Combinatorial Nullstellensatz that if $c_{P, \eta} \neq 0$, and $L$ is a list assignment of $G$ for which $|L(v)|=\eta(v)+1$, then $G$ is $L$-colorable. (Note that $P_{G}$ is a homogeneous polynomial, and all the monomials with nonzero coefficient are of highest degree.) In particular, if $c_{P_{G}, \eta} \neq 0$ and $\eta(v)<k$ for all $v \in V(G)$, then $G$ is $k$-choosable. Jensen and Toft [8] defined the Alon-Tarsi number of $G$ as

$$
A T(G)=\min \left\{k: c_{P_{G}, \eta} \neq 0 \text { for some } \eta \text { with } \eta(v)<k \text { for all } v \in V(G)\right\} .
$$

Thus for any graph $G, \operatorname{ch}(G) \leq A T(G)$. The following is the main result in [6].

Theorem 1.1 Every planar graph $G$ has a matching $M$ such that $A T(G-$ $M) \leq 4$. 
This theorem actually implies the online version of 1-defective 4-choosability of planar graphs. The online version of $d$-defective $k$-choosable is called $d$ defective $k$-paintable and is defined through a two-person game.

Given a graph $G$ and non-negative integers $k, d$, the $d$-defective $k$-painting game on $G$ is played by two players: Lister and Painter. Initially, each vertex has $k$ tokens and is uncolored. In each round, Lister selects a nonempty set $M$ of uncolored vertices and takes away one token from each vertex in $M$. Painter colors a subset $X$ of $M$ such that the induced subgraph $G[X]$ has maximum degree at most $d$. If at the end of a certain round, there is an uncolored vertex with no tokens left, then Lister wins. Otherwise, at the end of some round, all vertices are colored, Painter wins. We say $G$ is $d$-defective $k$-paintable if Painter has a winning strategy in the $d$-defective $k$-painting game. The 0 -defective $k$-painting game is also called the $k$-painting game, and we say $G$ is $k$-paintable if it is 0 -defective $k$-paintable. The paint number $\chi_{P}(G)$ of $G$ is the minimum $k$ such that $G$ is $k$-paintable.

It follows from the definition that $d$-defective $k$-paintable implies $d$ defective $k$-choosable. The converse is not true. Indeed, although every planar graph is 2-defective 3 -choosable, it was shown in [5] that there are planar graphs that are not 2-defective 3-paintable.

On the other hand, it was proved by Schauz [11] that for any graph $G$, $\chi_{P}(G) \leq A T(G)$. So for any graph $G, \operatorname{ch}(G) \leq \chi_{P}(G) \leq A T(G)$. Both gaps $\chi_{P}(G)-\operatorname{ch}(G)$ and $A T(G)-\chi_{P}(G)$ can be arbitrarily large [3]. Thus Theorem 1.1 implies that every planar graph is 1-defective 4-paintable. We observe that "having a matching $M$ so that $A T(G-M) \leq 4$ " is much stronger than "being 1-defective 4-paintable". One may compare this to the following results: It is shown in [5] that every planar graph is 3-defective 3 -paintable. However, as mentioned earlier, there are planar graphs $G$ such that for any subgraph $H$ of $G$ with $\Delta(H) \leq 3, G-E(H)$ is not 3-choosable [9] (and hence $A T(G-E(H)) \geq 4$ ).

In this paper, we are interested in the Alon-Tarsi number of some subgraphs of planar graphs without cycles of lengths 4 and $l$ for some $l \in$ $\{5,6,7\}$. We denote by $\mathcal{P}_{k, l}$ the family of planar graphs $G$ which contains no cycles of length $k$ or $l$. It was proved in [10] that for $l \in\{5,6,7\}$, every graph $G \in \mathcal{P}_{4, l}$ is 1 -defective 3 -choosable. We strengthen this result and prove that for $l \in\{5,6,7\}$, every graph $G \in \mathcal{P}_{4, l}$ has a matching $M$ such that $G-M$ has Alon-Tarsi number at most 3. As discussed above, this implies that for $l \in\{5,6,7\}$, every graph $G \in \mathcal{P}_{4, l}$ is 1 -defective 3-paintable.

For a plane graph $G$, we denote its vertex set, edge set and face set by $V(G), E(G)$ and $F(G)$, respectively. For a vertex $v, d_{G}(v)$ (or $d(v)$ for short) is the degree of $v$. A vertex $v$ is called a $k$-vertex (respectively, a $k^{+}$-vertex or a $k^{-}$-vertex) if $d(v)=k$ (respectively, $d(v) \geq k$ or $\left.d(v) \leq k\right)$. For $e=u v \in E(G)$, we say $e$ is an $(a, b)$-edge if $d(u)=a$ and $d(v)=b$. For $f \in F(G)$, we denote $f=\left[u_{1} u_{2} \cdots u_{n}\right]$ if $u_{1}, u_{2}, \cdots, u_{n}$ are the boundary 
vertices of $f$ in cyclic order. A 3 -face $\left[u_{1} u_{2} u_{3}\right]$ is a $\left(d_{1}, d_{2}, d_{3}\right)$-face if $d\left(u_{i}\right)=$ $d_{i}$ for $i=1,2,3$.

\section{The main result}

The following is the main result of this paper.

Theorem 2.1 For $l \in\{5,6,7\}$, every graph $G \in \mathcal{P}_{4, l}$ has a matching $M$ such that $A T(G-M) \leq 3$.

For the proof of Theorem 2.1, we use an alternate definition of AlonTarsi number. A digraph $D$ is Eulerian if $d_{D}^{+}(v)=d_{D}^{-}(v)$ for every vertex $v$. Note that an Eulerian digraph needs not be connected. In particular, a digraph with no arcs is an Eulerian digraph. Assume $G$ is a graph and $D$ is an orientation of $G$. Let $\mathcal{E}_{e}(D)$ (respectively, $\mathcal{E}_{o}(D)$ ) be the set of spanning Eulerian sub-digraphs of $D$ with an even (respectively, an odd) number of arcs. Let

$$
\operatorname{diff}(D)=\left|\mathcal{E}_{e}(D)\right|-\left|\mathcal{E}_{o}(D)\right| .
$$

An orientation $D$ of $G$ is Alon-Tarsi $(A T)$ if $\operatorname{diff}(D) \neq 0$. Alon and Tarsi [1] proved that if $D$ is an orientation of $G$, and $\eta\left(x_{v}\right)=d_{D}^{+}(v)$, then $c_{P_{G, \eta}}=$ $\pm \operatorname{diff}(D)$. Hence the Alon-Tarsi number of $G$ can be defined alternatively as

$$
A T(G)=\min \left\{k: G \text { has an AT orientation } D \text { with } \Delta_{D}^{+}(v)<k\right\} .
$$

The proof of Theorem 2.1 is by induction. For the purpose of using induction, instead of proving Theorem 2.1 directly, we shall prove a stronger and more technique result.

Definition 2.2 Assume $G$ is a plane graph and $v_{0}$ is a vertex on the boundary of $G$. A valid matching of $\left(G, v_{0}\right)$ is a matching $M$ which does not cover $v_{0}$.

Definition 2.3 Let $G$ be a plane graph and $v_{0}$ be a vertex on the boundary of $G$. An orientation $D$ of $G$ is good, if $D$ is $A T$ with $\Delta_{D}^{+}(v)<3$ and $d_{D}^{+}\left(v_{0}\right)=0$.

We shall prove the following result, which obviously implies Theorem

Theorem 2.4 Assume $l \in\{5,6,7\}, G \in \mathcal{P}_{4, l}$, and $v_{0}$ is a vertex on the boundary of $G$. Then $\left(G, v_{0}\right)$ has a valid matching $M$ such that there is a good orientation $D$ of $G-M$. 
The proof of Theorem 2.4 uses discharging method. We shall first describe a family of reducible configurations, i.e., configurations that cannot be contained in a minimum counterexample of Theorem 2.4. Then describe a discharging procedure that leads to a contradiction to the Euler's formula.

We shall frequently use the following lemma in the later proofs.

Lemma 2.5 Assume $D$ is a digraph with $V(D)=X_{1} \cup X_{2}$ and $X_{1} \cap X_{2}=\emptyset$. If all the arcs between $X_{1}$ and $X_{2}$ are from $X_{1}$ to $X_{2}$. Then $D$ is $A T$ if and only if $D\left[X_{1}\right]$ and $D\left[X_{2}\right]$ are both $A T$.

Proof. Denote by $D_{1}$ and $D_{2}$ the sub-digraphs $D\left[X_{1}\right]$ and $D\left[X_{2}\right]$ of $D$, respectively. Note that the set of arcs of an Eulerian digraph can be decomposed into arc disjoint union of directed cycles. Since all the arcs between $X_{1}$ and $X_{2}$ are from $X_{1}$ to $X_{2}$, and hence none of them is contained in a directed cycle, we conclude that none of these arcs is contained in an Eulerian sub-digraphs of $D$. Hence each Eulerian sub-digraph $H$ of $D$ is the arc disjoint union of an Eulerian sub-digraph $H_{1}$ of $D_{1}$ and an Eulerian sub-digraph $H_{2}$ of $D_{2}$. Now $H$ is even if and only if $H_{1}, H_{2}$ have the same parity. Hence $\left|\mathcal{E}_{e}(D)\right|=\left|\mathcal{E}_{e}\left(D_{1}\right)\right| \times\left|\mathcal{E}_{e}\left(D_{2}\right)\right|+\left|\mathcal{E}_{o}\left(D_{1}\right)\right| \times\left|\mathcal{E}_{o}\left(D_{2}\right)\right|$, $\left|\mathcal{E}_{o}(D)\right|=\left|\mathcal{E}_{e}\left(D_{1}\right)\right| \times\left|\mathcal{E}_{o}\left(D_{2}\right)\right|+\left|\mathcal{E}_{o}\left(D_{1}\right)\right| \times\left|\mathcal{E}_{e}\left(D_{2}\right)\right|$. This implies that $\operatorname{diff}(D)=\operatorname{diff}\left(D_{1}\right) \times \operatorname{diff}\left(D_{2}\right)$. Thus, $D$ is AT if and only if $D_{1}$ and $D_{2}$ are both AT.

Assume Theorem 2.4 is not true and $G$ is a counterexample with minimum number of vertices. Let $f_{0}$ denote the outer face of $G$.

Lemma 2.6 $G$ is 2-connected. Moreover, $d_{G}(v) \geq 3$ for all $v \in V(G)-$ $\left\{v_{0}\right\}$.

Proof. Assume $G$ is not 2-connected. Let $B$ be a block of $G$ that contains a unique cut vertex $z^{*}$ and does not contain $v_{0}$. Let $G_{1}=G-\left(B-\left\{z^{*}\right\}\right)$. By the minimality, $\left(G_{1}, v_{0}\right)$ has a valid matching $M_{1}$ and there is a good orientation $D_{1}$ of $G_{1}-M_{1},\left(B, z^{*}\right)$ has a valid matching $M_{2}$ and there is a good orientation $D_{2}$ of $B-M_{2}$. Let $M=M_{1} \cup M_{2}$ and $D=D_{1} \cup D_{2}$. Applying Lemma 2.5 (with $X_{1}=V(B)-\left\{z^{*}\right\}$ and $X_{2}=V\left(G_{1}\right)$ ), $D$ is AT. So $M$ is a valid matching of $\left(G, v_{0}\right)$, and $G-M$ has a good orientation, a contradiction.

For the moreover part, assume to the contrary that $v \in V(G)-\left\{v_{0}\right\}$ and $d_{G}(v) \leq 2$. By induction hypothesis, $G^{\prime}=G-\{v\}$ has a valid matching $M$ such that $G^{\prime}-M$ has a good orientation $D^{\prime}$. Extend $D^{\prime}$ to an orientation $D$ of $G-M$ in which $v$ is a source vertex. It is obvious that $D$ is a good orientation of $G-M$.

Lemma 2.7 $G-\left\{v_{0}\right\}$ does not contain two adjacent 3-vertices. 
Proof. Assume to the contrary that $u v \in E(G)$ with $d(u)=d(v)=3$ and $u, v \neq v_{0}$. Let $G^{*}=G-\{u, v\}$. Then $\left(G^{*}, v_{0}\right)$ has a valid matching $M^{*}$ such that there exists a good orientation $D^{*}$ of $G^{*}-M^{*}$. Let $M=M^{*} \cup\{u v\}$. Then $M$ is a valid matching of $\left(G, v_{0}\right)$. Extend $D^{*}$ to an orientation $D$ of $G-M$ in which $u, v$ are sources. Then $D$ is a good orientation of $G-M$.

Definition 2.8 A 3-face $f$ is called a minor triangle if $f$ is a $(3,4,4)$-face and $v_{0}$ is not on $f$. A 3-vertex $v$ is called a minor 3-vertex if $v$ is incident to a triangle and $v \neq v_{0}$.

Definition 2.9 A triangle chain in $G$ of length $k$ is a subgraph of $G-\left\{v_{0}\right\}$ consisting of vertices $w_{1}, w_{2}, \ldots, w_{k+1}, u_{1}, u_{2}, \ldots, u_{k}$ in which $\left[w_{i} w_{i+1} u_{i}\right]$ is $a(4,4,4)$-face for $i=1,2, \ldots, k$, as depicted in Figure 1(a). We denote by $T_{i}$ the triangle $\left[w_{i} w_{i+1} u_{i}\right]$ and denote such a triangle chain by $T_{1} T_{2} \ldots T_{k}$. For convenience, a single 4-vertex is a triangle chain with 0 triangles. We say a triangle $T$ intersects a triangle chain $T_{1} T_{2} \ldots T_{k}$, if $T$ has one common vertex with $T_{1}$.

Lemma 2.10 If a minor triangle $T_{0}$ intersects a triangle chain $T_{1} T_{2} \ldots T_{k}$, then no vertex of $T_{k}$ is adjacent to a 3-vertex, except possibly $v_{0}$. In particular, the $k=0$ case implies that no vertex of a minor triangle $T_{0}$ is adjacent to a 3-vertex $v \in V(G)-\left(V\left(T_{0}\right) \cup\left\{v_{0}\right\}\right)$.

Proof. Assume to the contrary that $T_{0}=\left[w_{0} w_{1} u_{0}\right]$ is a minor triangle that intersects a triangle chain $T_{1} T_{2} \ldots T_{k}$, with $T_{i}=\left[w_{i} w_{i+1} u_{i}\right](1 \leq i \leq k)$, and $w_{k+1}$ has a neighbour $x$ with $d(x)=3$, as in Figure 1(b). Assume $w_{0}$ is a 3-vertex. Let $X=\cup_{i=0}^{k} V\left(T_{i}\right) \cup\{x\}$ and $G^{\prime}=G-X$. By the minimality of $G,\left(G^{\prime}, v_{0}\right)$ contains a valid matching $M^{\prime}$ and there is a good orientation $D^{\prime}$ of $G^{\prime}-M^{\prime}$.

Let $M=M^{\prime} \cup\left\{w_{0} u_{0}, w_{1} u_{1}, \ldots, w_{k} u_{k}, w_{k+1} x\right\}$. Then $M$ is a valid matching of $\left(G, v_{0}\right)$. Let $D$ be an orientation of $G-M$ obtained from $D^{\prime}$ by adding $\operatorname{arcs}\left(w_{i}, w_{i+1}\right)$ and $\left(w_{i+1}, u_{i}\right)$ for $i=0,1, \ldots, k$, and all the edges between $X$ and $V-X$ are oriented from $X$ to $V-X$, as depicted in Figure 1(c). Since $D[X]$ is acyclic, $D[X]$ is AT. By Lemma 2.5, $D$ is AT. It is easy to see that $\Delta_{D}^{+}(v)<3$ and $d_{D}^{+}\left(v_{0}\right)=0$. Thus $D$ is a good orientation of $G-M$.

Lemma 2.11 If a triangle chain $T_{1} T_{2} \ldots T_{k}$ intersects a minor triangle $T_{0}$, then the distance between $T_{k}$ and another minor triangle is at least 2. In particular, the $k=0$ case implies that any two minor triangles have distance at least 2 .

Proof. Assume to the contrary that $T_{1} T_{2} \ldots T_{k}$ with $T_{i}=\left[w_{i} w_{i+1} u_{i}\right]$ $(1 \leq i \leq k)$ is a triangle chain that intersects a minor triangle $T_{0}=\left[w_{0} w_{1} u_{0}\right]$, and the distance between $T_{k}$ and another minor triangle $T_{0}^{\prime}=[x y z]$ with 
(a)

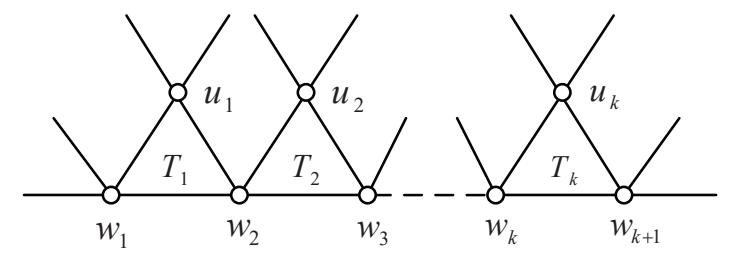

(b)

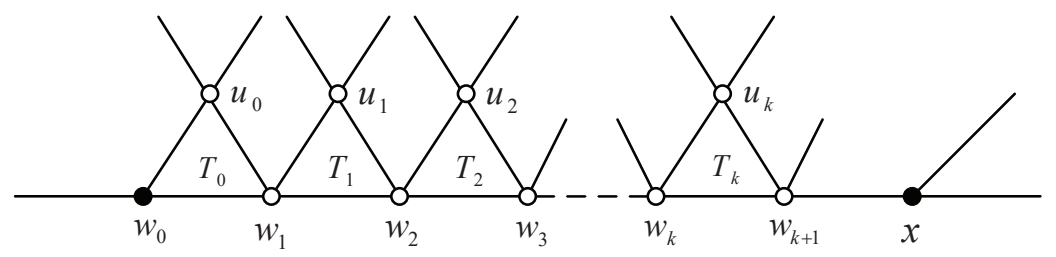

(c)

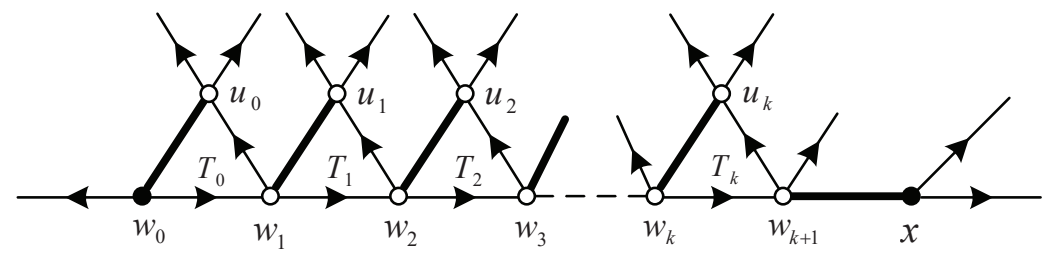

Figure 1: (a) A triangle chain. (b) The configuration in Lemma 2.10. (c) For the proof of Lemma 2.10, where a thick line is an edge in the matching $M$.

$d(x)=3$ is less than 2. By Lemma 2.10, we may assume $w_{k+1} y$ is a $(4,4)$ edge connecting $T_{k}$ and $T_{0}^{\prime}$, as in Figure 2(a). Let $X=\cup_{i=0}^{k} V\left(T_{i}\right) \cup V\left(T_{0}^{\prime}\right)$ and $G^{\prime}=G-X$. Then $\left(G^{\prime}, v_{0}\right)$ has a valid matching $M^{\prime}$ and there is a good orientation $D^{\prime}$ of $G^{\prime}-M^{\prime}$.

Let $M=M^{\prime} \cup\left\{w_{0} u_{0}, w_{1} u_{1}, \ldots, w_{k} u_{k}, w_{k+1} y, x z\right\}$. Then $M$ is a valid matching of $\left(G, v_{0}\right)$. Let $D$ be an orientation of $G-M$ obtained from $D^{\prime}$ by adding $\operatorname{arcs}(x, y),(y, z),\left(w_{i}, w_{i+1}\right)$ and $\left(w_{i+1}, u_{i}\right)$ for $i=0,1, \ldots, k$, and all the edges between $X$ and $V-X$ are oriented from $X$ to $V-X$, as in Figure 2(b). Obviously, $D[X]$ is acyclic, so $D[X]$ is AT. By Lemma 2.5, $D$ is AT. Additionally, $\Delta_{D}^{+}(v)<3$ and $d_{D}^{+}\left(v_{0}\right)=0$. That is to say, $D$ is a good orientation of $G-M$, a contradiction.

The remainder of the proofs use a discharging procedure. The initial charge ch is defined as: $\operatorname{ch}(x)=d(x)-4$ for $x \in V(G) \cup F(G)$. Applying equalities $\sum_{v \in V(G)} d(v)=2|E(G)|=\sum_{f \in F(G)} d(f)$ and Euler's formula $|V(G)|-$ $|E(G)|+|F(G)|=2$, we conclude that

$$
\sum_{x \in V(G) \cup F(G)} \operatorname{ch}(x)=-8 .
$$

In a discharging procedure, $\operatorname{ch}(x \rightarrow y)$ denotes the charge discharged 
(a)

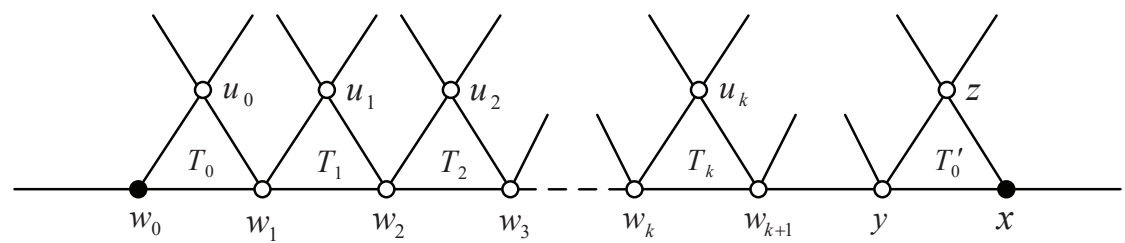

(b)

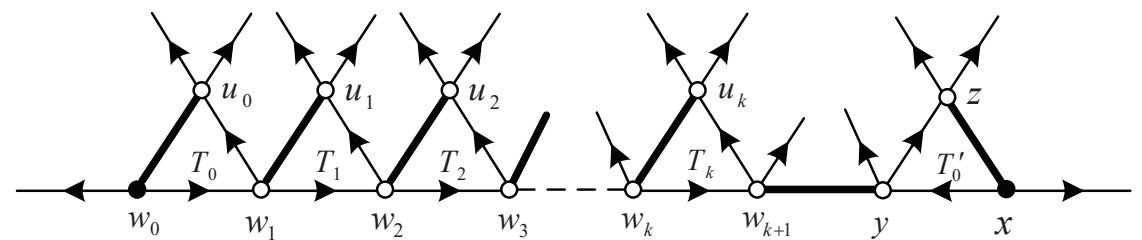

Figure 2: (a) The configuration in Lemma 2.11. (b) For the proof of Lemma 2.11 . where a thick line is an edge in the matching $M$.

from an element $x$ to another element $y, \operatorname{ch}(x \rightarrow)$ and $\operatorname{ch}(\rightarrow x)$ denote the charge totally discharged from or to $x$, respectively. The final charge $\operatorname{ch}^{*}(x)$ of $x \in V(G) \cup E(G)$ is defined as $\operatorname{ch}^{*}(x)=\operatorname{ch}(x)-\operatorname{ch}(x \rightarrow)+\operatorname{ch}(\rightarrow x)$. By applying appropriate discharging rules, we shall arrive at a final charge that $c h^{*}(x) \geq 0$ for all $x \in V(G) \cup F(G) \backslash\left\{v_{0}, f_{0}\right\}$, and $c h^{*}\left(v_{0}\right)+c h^{*}\left(f_{0}\right)>-8$. As the total charge does not change in the discharging process, this is a contradiction.

The discharging rules for graphs $G \in \mathcal{P}_{4, l}$ for $l \in\{5,6,7\}$ are different. We use three sections to discuss graphs $G \in \mathcal{P}_{4, l}$ for $l \in\{5,6,7\}$, respectively.

\section{Planar graphs without 4- and 5-cycles}

This section considers plane graphs without 4- and 5-cycles. We first derive more properties of a minimal counterexample $G$ to Theorem 2.4, where $G \in \mathcal{P}_{4,5}$.

Lemma 3.1 Assume $f$ is a 6-face of $G$ which is adjacent to five triangles, and none of the vertices in these triangles is $v_{0}$. If $f$ has one 3 -vertex, then there is at least one $5^{+}$-vertex on the five triangles.

Proof. Let $f=\left[v_{1} v_{2} v_{3} v_{4} v_{5} v_{6}\right], v_{1}$ be a 3 -vertex and $T_{i}=\left[v_{i} v_{i+1} u_{i}\right](i=$ $1,2, \ldots, 5)$ be the five triangles (see Figure 3(a)). Assume to the contrary that there is no $5^{+}$-vertex on $T_{i}$. By Lemma 2.10, we may assume all $v_{i+1}$ and $u_{i}$ are 4 -vertices for $i=1,2, \ldots, 5$. Let $X=\cup_{i=1}^{5} V\left(T_{i}\right)$ and $G^{\prime}=G-X$. Then $\left(G^{\prime}, v_{0}\right)$ has a valid matching $M^{\prime}$ and there is a good orientation $D^{\prime}$ of $G^{\prime}-M^{\prime}$.

Let $M=M^{\prime} \cup\left\{v_{1} u_{1}, v_{2} u_{2}, \ldots, v_{5} u_{5}\right\}$. Then $M$ is a valid matching of $\left(G, v_{0}\right)$. Let $D$ be the orientation of $G-M$ obtained from $D^{\prime}$ by adding arcs 


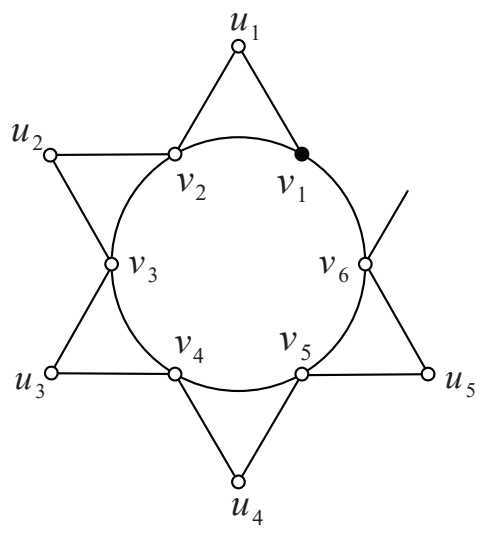

(a)

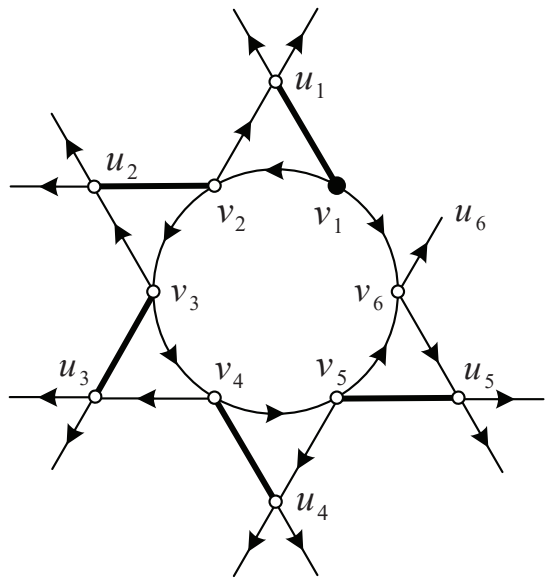

(b)

Figure 3: (a) The configuration in Lemma 3.1. (b) For the proof of Lemma 3.1. where a thick line is an edge in the matching $M$

$\left(v_{1}, v_{6}\right)$ and $\left(v_{i+1}, u_{i}\right),\left(v_{i}, v_{i+1}\right)$ for $i=1, \ldots, 5$, and all the edges between $X$ and $V-X$ are oriented from $X$ to $V-X$ (see Figure 3(b)). Clearly, $\Delta_{D}^{+}(v)<3$ and $D$ is AT by Lemma 2.5 , a contradiction.

The discharging rules are as follows:

R1 Assume $f \neq f_{0}$ is a 3 -face. Then each face adjacent to $f$ transfers $\frac{1}{3}$ charge to $f$.

$\mathrm{R} 2$ Assume $v \neq v_{0}$ is 3 -vertex. If $v$ is contained in a triangle, then each of the other two faces incident to $v$ transfers $\frac{1}{2}$ charge to $v$; otherwise each face incident to $v$ transfers $\frac{1}{3}$ charge to $v$.

R3 Assume $u \neq v_{0}$ is a $5^{+}$-vertex and $f \neq f_{0}$ is a 6 -face. If $f$ is adjacent to $s$ triangles that are incident to $u$, then $u$ transfers $\frac{s}{6}$ charge to $f$.

R4 $f_{0}$ transfers $\frac{1}{3}$ charge to each adjacent triangle, and $\frac{1}{2}$ charge to each incident 3-vertex $v \neq v_{0} . v_{0}$ transfers $\frac{1}{3}$ charge to each 6 -face $f \neq f_{0}$ which is either incident to $v_{0}$, or is not incident to $v_{0}$ but adjacent to a triangle $T$ which is incident to $v_{0}$.

Claim 3.2 If a 6-face $f$ has three minor 3-vertices, then $\operatorname{ch}(\rightarrow f) \geq \frac{1}{2}$.

Proof. Assume $f=\left[v_{1} v_{2} v_{3} v_{4} v_{5} v_{6}\right]$. By Lemma 2.7, we may assume that $v_{1}, v_{3}$ and $v_{5}$ are the three minor 3 -vertices. Then each of $v_{1}, v_{3}, v_{5}$ is incident to exactly one triangle. Hence at most two of the three triangles intersect each other. Thus we may assume that the three triangles adjacent to $f$ are either $T_{1}, T_{2}, T_{4}$, or $T_{1}, T_{3}, T_{5}$, where $T_{i}=\left[v_{i} v_{i+1} u_{i}\right]$. 
Case 1 The three triangles incident to $f$ are $T_{1}, T_{2}, T_{4}$.

If $v_{0}$ is a vertex of $f$ or $T_{1}, T_{2}$ or $T_{4}$, then $v_{0}$ transfers $\frac{1}{3}$ charge to $f$ by R4. By Lemma 2.10, at least one of the three triangles have a $5^{+}$-vertex $v \neq v_{0}$ which sends at least $\frac{1}{6}$ charge to $f$. So $\operatorname{ch}(\rightarrow f) \geq \frac{1}{3}+\frac{1}{6}=\frac{1}{2}$.

Assume $v_{0}$ is not a vertex of $f, T_{1}, T_{2}$ or $T_{4}$. By Lemma 2.10, either $v_{2}$ is a $5^{+}$-vertex or both of $u_{1}$ and $u_{2}$ are $5^{+}$-vertices. In both cases, $f$ receives $\frac{1}{3}$ charge in total from $v_{2}, u_{1}$ and $u_{2}$. Moreover, by Lemma 2.10, either $v_{4}$ or $u_{4}$ is a $5^{+}$-vertex, which transfers $\frac{1}{6}$ charge to $f$. Hence, $\operatorname{ch}(\rightarrow f) \geq \frac{1}{3}+\frac{1}{6}=\frac{1}{2}$. Case 2 The three triangles incident to $f$ are $T_{1}, T_{3}, T_{5}$.

By Lemma 2.10, each of the three triangles has either a $5^{+}$-vertex or $v_{0}$ which transfers at least $\frac{1}{6}$ charge to $f$. Thus, $\operatorname{ch}(\rightarrow f) \geq \frac{1}{2}$.

Claim 3.3 If a 6-face $f$ has two 3-vertices other than $v_{0}$ and is adjacent to four triangles, then $\operatorname{ch}(\rightarrow f) \geq \frac{1}{3}$.

Proof. Assume $f=\left[v_{1} v_{2} v_{3} v_{4} v_{5} v_{6}\right]$ and $T$ is a triangle adjacent to $f$. If $v_{0}$ is a vertex of $f$ or $T$, then $v_{0}$ transfers $\frac{1}{3}$ charge to $f$ by R4. Assume $v_{0}$ is neither a vertex of $f$ nor a vertex of any triangle $T$ adjacent to $f$.

By Lemma 2.7, we may assume that either $v_{1}$ and $v_{4}$ or $v_{1}$ and $v_{3}$ are the two 3 -vertices. For $i=1,2, \ldots, 6$, if $v_{i} v_{i+1}$ is contained in a triangle, then let $T_{i}=\left[v_{i} v_{i+1} u_{i}\right]$ be the triangle. We need to consider five cases.

Case 1 The four triangles incident to $f$ are $T_{1}, T_{2}, T_{3}, T_{5}$ while the two 3 -vertices are $v_{1}$ and $v_{4}$.

If at least one of $v_{2}$ and $v_{3}$ is a $5^{+}$-vertex, then by R3, $\operatorname{ch}(\rightarrow f) \geq \frac{1}{3}$. Assume both $d\left(v_{2}\right)$ and $d\left(v_{3}\right)$ are 4 -vertices. By Lemma 2.10 and Lemma 2.11 at least two of $u_{1}, u_{2}$ and $u_{3}$ are $5^{+}$-vertices each of which transfers $\frac{1}{6}$ charge to $f$. So $\operatorname{ch}(\rightarrow f) \geq \frac{1}{3}$.

Case 2 The four triangles incident to $f$ are $T_{1}, T_{2}, T_{4}, T_{5}$ while the two 3 -vertices are $v_{1}$ and $v_{4}$.

By Lemma 2.10 and R3, at least one of $u_{1}, u_{2}, v_{2}$ and $v_{3}$ is a $5^{+}$-vertex transferring at least $\frac{1}{6}$ charge to $f$. By symmetry, at least one of $u_{4}, u_{5}, v_{5}$ and $v_{6}$ transfers at least $\frac{1}{6}$ charge to $f$. Thus, we are done.

Case 3 The four triangles incident to $f$ are $T_{1}, T_{2}, T_{4}, T_{5}$ while the two 3 -vertices are $v_{1}$ and $v_{3}$.

If $v_{2}$ is a $5^{+}$-vertex, then $v_{2}$ transfers $\frac{1}{3}$ charge to $f$ by R3. Assume $v_{2}$ is a 4 -vertex. By Lemma 2.10, both $u_{1}$ and $u_{2}$ are $5^{+}$-vertices each of which transfers $\frac{1}{6}$ charge to $f$.

Case 4 The four triangles incident to $f$ are $T_{1}, T_{3}, T_{4}, T_{5}$ while the two 3 -vertices are $v_{1}$ and $v_{3}$.

By Lemma 2.10, at least one of $v_{2}$ and $u_{1}$ is a $5^{+}$-vertex transferring $\frac{1}{6}$ charge to $f$. Moreover, using Lemma 2.10 again, at least one of $v_{4}, v_{5}, v_{6}$, $u_{3}, u_{4}$ and $u_{5}$ is a $5^{+}$-vertex transferring at least $\frac{1}{6}$ to $f$. So $\operatorname{ch}(\rightarrow f) \geq \frac{1}{3}$.

Case 5 The four triangles incident to $f$ are $T_{3}, T_{4}, T_{5}, T_{6}$ while the two 3 -vertices are $v_{1}$ and $v_{3}$. 
If one of $v_{4}, v_{5}$ and $v_{6}$ is a $5^{+}$-vertex, then such a $5^{+}$-vertex sends $\frac{1}{3}$ charge to $f$ by R3. Assume all of $v_{4}, v_{5}$ and $v_{6}$ are 4 -vertices. Then at least two of $u_{3}, u_{4}, u_{5}$ and $u_{6}$ are $5^{+}$-vertices each sending $\frac{1}{6}$ to $f$. Otherwise, it will contradict to Lemma 2.10 or Lemma 2.11. Again $\operatorname{ch}(\rightarrow f) \geq \frac{1}{3}$.

- Check charge on vertices $v \neq v_{0}$

Let $v$ be a 3 -vertex. By $\mathrm{R} 2, v$ gets 1 from incident $6^{+}$-faces. That is $\operatorname{ch}^{*}(v)=\operatorname{ch}(v)-\operatorname{ch}(v \rightarrow)+\operatorname{ch}(\rightarrow v)=-1-0+1=0$.

Let $v$ be a 4 -vertex. $\operatorname{ch}^{*}(v)=\operatorname{ch}(v)=0$.

Let $v$ be a $5^{+}$-vertex. By R3, $v$ only transfers charge to 6 -faces that are adjacent to a triangle incident to $v$. Assume $v$ is incident with $t$ triangles, then $0<t \leq\left\lfloor\frac{d(v)}{2}\right\rfloor$. Each triangle incident with $v$ is adjacent to at most three 6 -faces, and $v$ transfers $\frac{1}{6}$ to each of the three 6 -faces (note that if a 6 -face $f$ is adjacent to two triangles that are incident to $v$, then $v$ transfers $2 \times \frac{1}{6}$ charges to $\left.f\right)$. Hence $v$ sends out at most $\frac{1}{2} t$ charge. So we have $\operatorname{ch}^{*}(v)=\operatorname{ch}(v)-\operatorname{ch}(v \rightarrow) \geq d(v)-4-\frac{1}{2} t \geq d(v)-4-\frac{1}{2} \times\left\lfloor\frac{d(v)}{2}\right\rfloor \geq 0$.

- Check charge on faces $f \neq f_{0}$

Let $f$ be a 3 -face. R1 guarantees $c h^{*}(f) \geq 0$.

Let $f$ be a 6 -face. Assume that $f$ has $s$-vertices other than $v_{0}$. Then $s \leq 3$ by Lemma 2.7, and $f$ is adjacent to at most $(6-s)$ triangles.

If $s=0$, then $f$ sends out at most $\frac{1}{3}$ to each adjacent triangle, and hence $\operatorname{ch}(f \rightarrow) \leq \frac{1}{3} \times 6=2$ and $c h^{*}(f) \geq 0$.

Assume $s=3$. If $f$ is adjacent to at most two triangles, then $f$ has at most two minor 3 -vertices. So $\operatorname{ch}(f \rightarrow) \leq \frac{1}{2} \times 2+\frac{1}{3}+\frac{1}{3} \times 2=2$ and $c h^{*}(f) \geq 0$. Assume $f$ is adjacent to three triangles, then all these three 3 -vertices are minor. By Claim 3.2, we have $c^{*}(f)=d(f)-4-\operatorname{ch}(f \rightarrow$ )$+\operatorname{ch}(\rightarrow f) \geq 2-\left(\frac{1}{2} \times 3+\frac{1}{3} \times 3\right)+\frac{1}{2}=0$.

Assume $s=2$. If $f$ is adjacent to at most three triangle, then $\operatorname{ch}(f \rightarrow) \leq$ $\frac{1}{2} \times 2+\frac{1}{3} \times 3=2$ and $c h^{*}(f) \geq 0$. If $f$ is adjacent to four triangles, then by Claim 3.3. $\operatorname{ch}^{*}(f)=d(f)-4-\operatorname{ch}(f \rightarrow)+\operatorname{ch}(\rightarrow f) \geq 2-\left(\frac{1}{2} \times 2+\frac{1}{3} \times 4\right)+\frac{1}{3}=0$.

Assume $s=1$. If $f$ is adjacent to at most four triangles, then $\operatorname{ch}(f \rightarrow$ )$\leq \frac{1}{2}+\frac{1}{3} \times 4=\frac{11}{6}<2$. Assume $f$ is adjacent to five triangles. Then $\operatorname{ch}(f \rightarrow)=\frac{1}{2}+\frac{1}{3} \times 5=\frac{13}{6}$. On the other hand, either at least one vertex of the five triangles is a $5^{+}$-vertex transferring $\frac{1}{6}$ charge to $f$ by Lemma 3.1. or $v_{0}$ is a vertex of the five triangles transferring $\frac{1}{3}$ to $f$ by R4. Hence $c h^{*}(f) \geq 2-\frac{13}{6}+\frac{1}{6}=0$.

Let $f$ be a $7^{+}$-face. Assume $f$ has $s$-vertices other than $v_{0}$, then $s \leq\left\lfloor\frac{d(f)}{2}\right\rfloor$ and $f$ is adjacent to at most $(d(f)-s)$ triangles. Hence $c h^{*}(f)=$ $d(f)-4-\left[\frac{1}{2} \times s+\frac{1}{3} \times(d(f)-s)\right]=\frac{2}{3} d(f)-\frac{1}{6} s-4 \geq\left(\frac{2}{3}-\frac{1}{12}\right) d(f)-4>0$.

- Check charge on $f_{0}$ and $v_{0}$

By R4, it is clear that $v_{0}$ transfers at most $\left(d\left(v_{0}\right)-1\right) \times \frac{1}{3}$ charge to others. That is, $c h^{*}\left(v_{0}\right) \geq d\left(v_{0}\right)-4-\left(d\left(v_{0}\right)-1\right) \times \frac{1}{3}=\frac{2}{3} d\left(v_{0}\right)-\frac{11}{3} \geq-\frac{7}{3}$ (as $d\left(v_{0}\right) \geq 2$ ). 
Since $f_{0}$ is incident with at most $\left\lfloor\frac{d\left(f_{0}\right)}{2}\right\rfloor 3$-vertices each getting $\frac{1}{2}$ charge from it, and $f_{0}$ is adjacent to at most $d\left(f_{0}\right)$ triangles each getting $\frac{1}{3}$ charge from it. We have $c h^{*}\left(f_{0}\right) \geq d\left(f_{0}\right)-4-\frac{1}{2}\left\lfloor\frac{d\left(f_{0}\right)}{2}\right\rfloor-\frac{1}{3} d\left(f_{0}\right) \geq \frac{5}{12} d\left(f_{0}\right)-4 \geq-\frac{11}{4}$.

Consequently, we obtain the following contradiction, and the proof is complete.

$$
0 \leq \sum_{x \in V \cup F \backslash\left\{v_{0}, f_{0}\right\}} c h^{*}(x)=-8-c h^{*}\left(v_{0}\right)-c h^{*}\left(f_{0}\right) \leq-\frac{35}{12} .
$$

\section{Planar graphs without 4- and 6-cycles}

This section shows plane graph without 4- and 6-cycles. We list our discharging rules as follows:

R1 Assume $f \neq f_{0}$ is a 3 -face. Then each face adjacent to $f$ transfers $\frac{1}{3}$ charge to $f$.

$\mathrm{R} 2$ Assume $v \neq v_{0}$ is 3 -vertex. If $v$ is contained in a triangle, then each of the other two faces incident to $v$ transfers $\frac{1}{2}$ charge to $v$; otherwise each face incident to $v$ transfers $\frac{1}{3}$ charge to $v$.

R3 $f_{0}$ transfers $\frac{1}{3}$ charge to each adjacent triangle, and $\frac{1}{2}$ charge to each incident 3 -vertex $v \neq v_{0}$.

- Check charge on vertices $v \neq v_{0}$

For $d(v)=3, \mathrm{R} 2$ ensures that the final charge of $v$ is non-negative.

For $d(v) \geq 4$, no transference on $v$, we have $\operatorname{ch}^{*}(v)=\operatorname{ch}(v) \geq 0$.

- Check charge on faces $f \neq f_{0}$

Let $f$ be a 3 -face. R1 guarantees $c h(\rightarrow f)=1$. So $c h^{*}(f)=-1+1=0$.

Let $f$ be a 5 -face. Since $G$ does not contain 6 -cycle, $f$ is not adjacent to any triangle. Thus $f$ only discharges to the non-minor 3 -vertices each of which gets $\frac{1}{3}$ charge from $f$. On the other hand, $f$ is incident with at most two such 3 -vertices by Lemma 2.7. It concludes that $c h^{*}(f)=1-\frac{1}{3} \times 2>0$.

Let $f$ be a $7^{+}$-face. Assume $f$ is incident with $s 3$-vertices besides $v_{0}$. Then by Lemma 2.7, $s \leq\left\lfloor\frac{d(f)}{2}\right\rfloor$. By R1 and R2, $f$ transfers at most $\frac{1}{2} s$ to 3 -vertices and $(d(f)-s) \times \frac{1}{3}$ to triangles. Hence, we have $c h^{*}(f) \geq$ $d(f)-4-\frac{1}{2} s-\frac{1}{3}(d(f)-s)=\frac{2}{3} d(f)-\frac{1}{6} s-4 \geq \frac{7}{12} d(f)-4>0$.

- Check charge on $f_{0}$ and $v_{0}$

It is obvious that $\operatorname{ch}^{*}\left(v_{0}\right)=\operatorname{ch}\left(v_{0}\right)=d\left(v_{0}\right)-4 \geq-2$.

Since $f_{0}$ is incident with at most $\left\lfloor\frac{d\left(f_{0}\right)}{2}\right\rfloor 3$-vertices each getting $\frac{1}{2}$ charge from it, and $f_{0}$ is adjacent to at most $d\left(f_{0}\right)$ triangles each getting $\frac{1}{3}$ charge from it. We have $c^{*}\left(f_{0}\right) \geq d\left(f_{0}\right)-4-\frac{1}{2}\left\lfloor\frac{d\left(f_{0}\right)}{2}\right\rfloor-\frac{1}{3} d\left(f_{0}\right) \geq \frac{5}{12} d\left(f_{0}\right)-4 \geq-\frac{11}{4}$. 
Consequently, we obtain the following contradiction, and the proof is complete.

$$
0 \leq \sum_{x \in V \cup F \backslash\left\{v_{0}, f_{0}\right\}} c h^{*}(x)=-8-c h^{*}\left(f_{0}\right)-c h^{*}\left(v_{0}\right) \leq-\frac{13}{4} .
$$

\section{Planar graphs without 4- and 7-cycles}

In this section, we consider plane graphs without 4- and 7-cycles. First we derive more properties of a minimal counterexample $G$ to Theorem 2.4 for $G \in \mathcal{P}_{4,7}$.

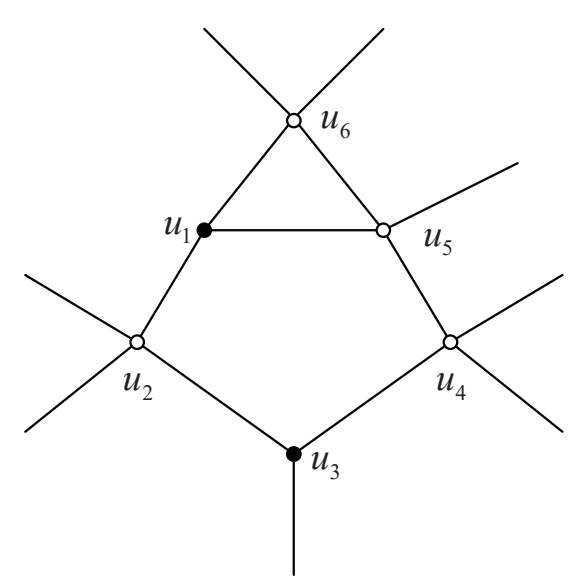

(a)

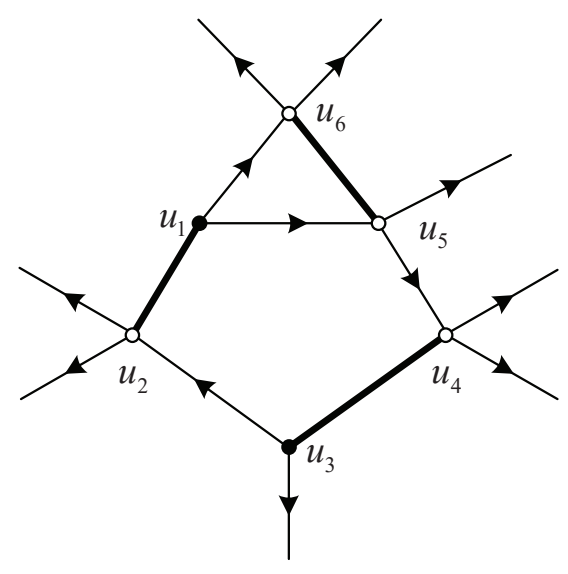

(b)

Figure 4: (a) A special 5-cycle and an adjacent triangle. (b) For the proof of Lemma 5.2 , where a thick line is an edge in the matching $M$.

Definition 5.1 A 5-cycle $f=\left[u_{1} u_{2} u_{3} u_{4} u_{5}\right]$ is called special if it is adjacent to a triangle $T=\left[u_{1} u_{5} u_{6}\right]$ with $u_{i} \neq v_{0}(i=1,2, \ldots, 6)$, and all the vertices are 4-vertices except that $u_{1}$ and $u_{3}$ are 3-vertices, as depicted in Figure 4(a).

Lemma 5.2 G has no special 5-cycle.

Proof. Assume $f=\left[u_{1} u_{2} u_{3} u_{4} u_{5}\right]$ is a special 5-cycle and $T=\left[u_{1} u_{5} u_{6}\right]$ is a triangle adjacent to $f$, where $d\left(u_{1}\right)=d\left(u_{3}\right)=3$ and $d\left(u_{i}\right)=4$ for $i=2,4,5,6$. Let $X=\left\{u_{1}, u_{2}, \ldots, u_{6}\right\}$ and $G^{\prime}=G-X$. Then, by the minimality, $\left(G^{\prime}, v_{0}\right)$ has a valid matching $M^{\prime}$ and there is a good orientation $D^{\prime}$ of $G^{\prime}-M^{\prime}$.

Let $M=M^{\prime} \cup\left\{u_{1} u_{2}, u_{3} u_{4}, u_{5} u_{6}\right\}$, then $M$ is a valid matching of $\left(G, v_{0}\right)$. Let $D$ be an orientation of $G$ obtained from $D^{\prime}$ by adding $\operatorname{arcs}\left(u_{1}, u_{6}\right)$, 
$\left(u_{1}, u_{5}\right),\left(u_{5}, u_{4}\right)$ and $\left(u_{3}, u_{2}\right)$, and all the edges between $X$ and $V-X$ are oriented from $X$ to $V-X$, as depicted in Figure 4(b). It is obvious that $D[X]$ is AT. Then, by Lemma 2.5, $D$ is AT. As $\Delta_{D}^{+}(v)<3$ and $d_{D}^{+}\left(v_{0}\right)=0$, $D$ is a good orientation of $G-M$, a contradiction.

The discharging rules are defined as follows:

R1 Assume $f \neq f_{0}$ is a 3 -face. Then each face adjacent to $f$ transfers $\frac{1}{3}$ charge to $f$.

$\mathrm{R} 2$ Assume $v \neq v_{0}$ is 3 -vertex. If $v$ is contained in a triangle, then each of the other two faces incident to $v$ transfers $\frac{1}{2}$ charge to $v$; otherwise each face incident to $v$ transfers $\frac{1}{3}$ charge to $v$.

R3 Assume $u \neq v_{0}$ is a $5^{+}$-vertex and $f \neq f_{0}$ is a 5 -face. Then $u$ transfers $\frac{1}{6}$ charge to $f$ either $f$ is incident to $u$, or $f$ is not incident to $u$ but adjacent to a triangle which is incident to $u$.

R4 $f_{0}$ transfers $\frac{1}{3}$ charge to each adjacent triangle, and $\frac{1}{2}$ charge to each incident 3-vertex $v \neq v_{0} . v_{0}$ transfers $\frac{1}{3}$ charge to each 5 -face $f \neq f_{0}$ which is either incident to $v_{0}$, or is not incident to $v_{0}$ but adjacent to a triangle $T$ which is incident to $v_{0}$.

- Check charge on vertices $v \neq v_{0}$

Let $v$ be a 3 -vertex. By $\mathrm{R} 2, c h^{*}(v) \geq 0$.

Let $v$ be a 4 -vertex. We have $c h^{*}(v)=\operatorname{ch}(v)=0$.

Let $v$ be a $5^{+}$-vertex. By $\mathrm{R} 3, v$ transfers at most $\frac{1}{6} \times d(v)$ charge to 5 -faces. It follows that $\operatorname{ch}^{*}(v) \geq d(v)-4-\frac{1}{6} d(v)=\frac{5}{6} d(v)-4>0$.

- Check charge on faces $f \neq f_{0}$

Let $f$ be a 3 -face. R1 guarantees $c h^{*}(f) \geq 0$.

Let $f$ be a 5 -face. By Lemma 2.7, $f$ has at most two 3-vertices other than $v_{0}$. Since $G$ has no 7-cycle, $f$ is adjacent to at most one triangle. Namely, $f$ has at most one minor 3 -vertex. If $f$ has at most one 3 -vertex other than $v_{0}$, then $\operatorname{ch}(f \rightarrow) \leq \frac{1}{2}+\frac{1}{3}<1$. Assume $f$ has two 3 -vertices other than $v_{0}$.

Firstly, if $f$ does not have any minor 3 -vertex, then $f$ transfers at most $\frac{1}{3}$ charge to the unique triangle and $\frac{1}{3} \times 2$ to the non-minor 3 -vertices. That is, $c h^{*}(f)=\operatorname{ch}(f)-\operatorname{ch}(f \rightarrow) \geq 1-1=0$.

Assume $f$ has a minor 3 -vertex. Assume $f=\left[v_{1} v_{2} v_{3} v_{4} v_{5}\right]$ with $d\left(v_{1}\right)=3$ and $T=\left[v_{1} v_{2} v_{6}\right]$. In this case, $\operatorname{ch}(f \rightarrow)=\frac{1}{2}+\frac{1}{3}+\frac{1}{3}=\frac{7}{6}$. If one of $v_{i}$ $(i=2,3, \ldots, 6)$ is $v_{0}$ or a $5^{+}$-vertex, then such $v_{i}$ transfers at least $\frac{1}{6}$ charge to $f$ by R4 and R3. Thus, $\operatorname{ch}^{*}(f)=\operatorname{ch}(f)-\operatorname{ch}(f \rightarrow)+\operatorname{ch}(\rightarrow f) \geq 1-\frac{7}{6}+\frac{1}{6} \geq 0$. Assume $f$ and $T$ does not contain $v_{0}$ and $5^{+}$-vertex. By Lemma 2.7 and Lemma 2.10, another 3 -vertex must be $v_{4}$. Thus, there is a special 5 -cycle in $G$, contradicting to Lemma 5.2 . 
Let $f$ be a 6 -face. By Lemma 2.7, $f$ has at most three 3 -vertice other than $v_{0}$. Since $G$ has no 4 - and 7 -cycles, $f$ is adjacent to at most one triangle $T$ which shares two common edges with $f$. If $f$ is not adjacent to any triangle, $f$ only sends charge to non-minor 3 -vertices each getting $\frac{1}{3}$ from $f$. Hence, $c h^{*}(f) \geq d(f)-4-\frac{1}{3} \times 3>0$. If $f$ is adjacent to one triangle $T$ which shares two common edges with $f$, then $f$ has at most one minor 3 -vertex. Thus, $c h^{*}(f) \geq d(f)-4-\frac{1}{2}-\frac{1}{3} \times 2-\frac{1}{3}>0$.

Let $f$ be a $8^{+}$-face. If $f$ is incident with $s 3$-vertices other than $v_{0}$ where $0 \leq s \leq\left\lfloor\frac{d(f)}{2}\right\rfloor$. Then $f$ transfers at most $\frac{1}{2} \times s$ charge to 3 -vertices and $(d(f)-s) \times \frac{1}{3}$ charge to triangles. Hence, we have $c^{*}(f) \geq d(f)-4-\frac{1}{2} s-$ $\frac{1}{3}(d(f)-s)=\frac{2}{3} d(f)-\frac{1}{6} s-4 \geq \frac{7}{12} d(f)-4>0$.

- Check charge on $f_{0}$ and $v_{0}$

For this checking procedure is the same as the last part in Section 3, we omit the details. That is $c h^{*}\left(v_{0}\right) \geq-\frac{7}{3}$ and $c h^{*}\left(f_{0}\right) \geq-\frac{11}{4}$.

Thus, we will have $0 \leq \sum_{x \in V \cup F \backslash\left\{v_{0}, f_{0}\right\}} c h^{*}(x)=-8-c h^{*}\left(v_{0}\right)-c h^{*}\left(f_{0}\right) \leq$ $-\frac{35}{12}$, a contradiction.

\section{References}

[1] N. Alon and M. Tarsi, Colorings and orientations of graphs, Combinatorica 12 (2) (1992) 125-134.

[2] W. Cushing and H. A. Kierstead, Planar graphs are 1-relaxed, 4choosable, European J. Combin. 31(5) (2010) 1385-1397.

[3] L. Duraj, G. Gutowski and J. Kozik, Chip games and paintability, Electron. J. Combin. 23 (2016), no. 3, Paper 3.3, 12 pp.

[4] N. Eaton and T. Hall, Defective list colorings of planar graphs, Bull. Inst. Combin. Appl. 25(1999) 79-87.

[5] G. Gutowski, M. Han, T. Krawczyk and X. Zhu, Defective 3-paintability of planar graphs, Electron. J. Combin. 25 (2018), no. 2, Paper 2.34, 20 pp.

[6] J. Grytczuk and X. Zhu, The Alon-Tarsi number of a planar graph minus a matching, arXiv:1811.12012.

[7] M. Han and X. Zhu, Locally planar graphs are 2-defective 4-paintable, European J. Combin. 54 (2016) 35-50.

[8] T. Jensen and B. Toft, Graph Coloring Problems, Wiley, New York, 1995.

[9] R. Kim, S-J. Kim, X. Zhu. The Alon-Tarsi number of subgraphs of a planar graph, manuscript. 
[10] K-W. Lih, Z. Song, W. Wang and K. Zhang, A note on list improper coloring planar graphs, Appl. Math. Lett. 14 (2001) 269-273.

[11] U. Schauz, Flexible color lists in Alon and Tarsi's theorem, and time scheduling with unreliable participants, Electron. J. Combin. 17 (2010):R13:1-18.

[12] R. Škrekovski, List improper colourings of planar graphs, Combin. Probab. Comput. 8 (1999) 293-299. 